\title{
Markers of vascular differentiation, proliferation and tissue remodeling in juvenile nasopharyngeal angiofibromas
}

\author{
SUELY NONOGAKI ${ }^{1}$, HELOISA G.A. CAMPOS ${ }^{2}$, OSSAMU BUTUGAN ${ }^{3}$, FERNANDO A. SOARES ${ }^{2}$, \\ FLÁVIA REGINA ROTEA MANGONE ${ }^{4}$, HUMBERTO TORLONI ${ }^{2}$ and M. MITZI BRENTANI ${ }^{4}$
}

\author{
${ }^{1}$ Instituto Adolfo Lutz, Central, Divisão de Patologia, Laboratório de Imuno-histoquímica, CEP 01246-902; \\ ${ }^{2}$ Hospital A.C. Camargo, CEP 01509-010; ${ }^{3}$ Departamento de Oftalmologia e Otorrinolaringologia, Faculdade de Medicina \\ da Universidade de São Paulo, Cerqueira César CEP 05403-010; ${ }^{4}$ Departamento de Radiologia e Oncologia, \\ Disciplina de Oncologia, Faculdade de Medicina da Universidade de São Paulo (LIM-24), CEP 01246-903, São Paulo, Brasil
}

Received June 18, 2010; Accepted July 21, 2010

DOI: $10.3892 / \mathrm{etm} .2010 .141$

\begin{abstract}
Juvenile nasopharingeal angiofibroma (JNA) is a histologically benign locally aggressive tumor characterized by irregular vessels embedded in a fibrous stroma. Excessive vascularity results in bleeding complications, and the inhibition of angiogenesis is a promising strategy for managing extensive JNA tumors. To better characterize the endothelial components of JNA, we aimed to evaluate markers of vascular differentiation and proliferation, such as friend leukemia integration-1 (FLI-1) and endoglin, lymphatic markers, including podoplanin and vascular endothelial growth factor receptor 3 (VEGFR3) and its cognate ligand VEGFC, GLUT-1, a diagnostic marker that discriminates between hemangiomas and vascular malformations, and two markers of tissue remodeling, stromelysin 3 (ST3) and secreted acid protein rich in cysteine (SPARC). Antigens were assessed immunohistochemically in vessels and stromal cells of JNA archival cases $(n=22)$. JNA endothelial cells were positive for endoglin, VEGFC and FLI-1, whereas podoplanin and VEGFR3 were negative in all cases. Both endothelial cells and fibroblasts stained for ST3 and SPARC. GLUT-1 was investigated in JNA cases, in infantile hemangiomas $(n=123)$ and in vascular malformations $(\mathrm{n}=135)$ as controls. JNAs and vascular malformations were GLUT-1-negative, while hemangiomas showed positive staining. The presence of markers of endothelial differentiation and proliferation highlighted the hyper-proliferative state of JNA vessels. The absence of podoplanin and VEGFR3 underscores their blood endothelial
\end{abstract}

Correspondence to: Dr M. Mitzi Brentani, Departamento de Radiologia e Oncologia, Disciplina de Oncologia, Faculdade de Medicina da Universidade de São Paulo (LIM-24), Av. Dr. Arnaldo 455, CEP 01246-903, São Paulo, SP, Brasil

E-mail: mbrentani@lim24.fm.usp.br

Key words: juvenile nasopharingeal angiofibroma, endoglin, friend leukemia integration-1, podoplanin, vascular endothelial growth factor receptor 3, GLUT-1, stromelysin 3, secreted acid protein rich in cysteine cell characteristic. The absence of GLUT-1 discriminates JNAs from hemangiomas. ST3 and SPARC up-regulation in endothelial cells and fibroblasts may contribute to a compensatory signaling for controlling angiogenesis. Some of these markers may eventually serve as therapeutic targets. Our results may aid in the understanding of JNA pathophysiology.

\section{Introduction}

Juvenile nasopharyngeal angiofibroma (JNA) is a rare benign neoplasm characterized by proliferating irregular vascular channels within a fibrous stroma composed of spindle cells in a dense collagen matrix $(1,2)$. Despite being classified as benign tumors, JNAs often exhibit an aggressive growth pattern with possible intracranial spreading. These features have stimulated numerous theories on tumor origin, focusing on the vascular or the stromal component (3). Nevertheless, the implicated neoplastic cell responsible for sustained growth remains a matter of debate $(1,3,4)$.

Recent studies based on vascular irregularities (thickness of the vessel walls, discontinuous vascular basal laminae and focal lack of pericytes) have led to the hypothesis that JNA is a vasoproliferative malformation $(2,3)$. This suggestion of a vascular tumor origin is supported by an embryological explanation, based on the involvement of plexus remnants deriving from incomplete regression of the first branchial arch artery, which were incorporated into the vascular tumor component $(3,4)$.

The presence of proteins such as merosin (laminin $\alpha 2$ ) and collagens type VI and 1A2, which were recently described as being highly expressed in JNA vessels, is suggestive of vessels in an early developmental vascular differentiation state (5-7). Notably, merosin and type VI collagen were also elevated in several types of cancer and in proliferative hemangiomas. The latter are vascular lesions characterized by abnormal excessive endothelial cell proliferation (5-9).

A series of immunohistochemical studies have been performed to analyze angiogenic activators and vessel growth promoting factors in JNA samples. Vascular markers, such as CD31, CD34 and von Willebrand factor (vWF), were previously 
documented in JNAs. However, such pan endothelium biomarkers did not discriminate between vascular and lymphatic cells, and some are not restricted to blood vessels (10). TGF $\beta 1$, FGF, vascular endothelial growth factor (VEGF A) and its receptors, VEGFR1 (Flt-1) and VEGFR2 (KDR), were also reported to be expressed in JNA endothelial cells (11-19). Of note, several of these growth factors were also elevated in both hemangiomas and in tumor-associated blood vessels $(8,20)$.

Thus, is would appear that JNA vessels exhibit malformative and endothelial proliferative features, as well as neoplastic markers, although they maintain benign characteristics. Similar aspects of angiogenesis in vascular anomalies and tumors were recently discussed (4).

Given that the most conspicuous feature of JNAs is the endothelial proliferative state, inhibition of angiogenesis is thought to be a promising strategy for their treatment. Our research has focused on identifying other markers that may provide additional information regarding the abnormal vascular component of JNAs. The first step in the present study was to evaluate the expression of endothelial markers involved in cell differentiation and cell growth, such as friend leukemia integration-1 (Fli-1) and endoglin. Fli-1 is a nuclear transcription factor that is considered to be a very reliable marker of endothelial differentiation. Fli-1 was reported to be involved in cellular proliferation and tumorigenesis, as well as in the suppression of the expression of genes such as $\mathrm{Rb}$ and $\mathrm{Bcl}-2$ protein $(21,22)$. Endoglin (CD105) is a transmembranic glycoprotein that is a marker of activated endothelium; its vascular expression is limited to proliferating endothelial cells, with no expression in normal vessels (23). To investigate the vascular differentiation state of JNA endothelial cells, specific markers of lymphatic vessels, such as podoplanin and VEGFR3 and its ligand VEGFC $(24,25)$, were also analyzed. The erythrocyte transporter (GLUT-1) was described in fetal endothelial cells (26) in solid tumors with enhanced glycolitic demand (27), and is a universal trait of hemangiomas, discriminating these tumors from vascular malformations (28). Thus, we evaluated the immunohistochemical expression of GLUT-1 in JNAs and compared its expression to that displayed by hemangiomas and vascular malformations used as the control.

Studies focused on differences in gene expression in endothelial cells derived from tumor tissues have revealed the enrichment of genes involved in tissue remodeling, a hallmark of post developmental angiogenesis, such as stromelysin 3 (ST3) and secreted acid protein rich in cysteine (SPARC; or osteonectin). These are up-regulated in a number of pathological processes (29-31). ST3 (MMP11) is a member of the metalloprotease (MMP) family and is an established connective tissue-derived factor associated with embryogenesis, wound healing and tissue involution (31). SPARC is a matricellular glycoprotein that has counter adhesive and antiproliferative functions and is associated with developing vessels and tumor blood vessels (32). Its importance in the angiogenic events in JNAs has been previously hypothesized (33). Thus, the expression of ST3 and SPARC was investigated in the present study.

\section{Materials and methods}

Case selection. Twenty-two patients with JNA who underwent surgery between 2000 and 2006 at the Hospital das Clínicas da Faculdade de Medicina da Universidade de São Paulo were selected for this study. All patients were male and between 12 and 25 years of age at the time of surgery (median 16 years). Since the study was retrospective, informed patient consent was waived. However, any form of patient identification was avoided. The study was approved by the Ethical Committee for Research Projects of the Hospital das Clínicas da Faculdade de Medicina da Universidade de São Paulo.

The tumors were staged using the Fisch system and ranged from stage II to IIIa. Recurrence was observed in $20 \%$ of the cases. Frozen tumor samples were obtained from 18 of the patients with JNAs; samples of paired turbinate tissues were obtained from 15 of the 18 patients and used for ST3 mRNA determination by Northern blot analysis.

In addition, 124 cases of proliferative hemangioma and 135 cases of vascular malformation excised at the Hospital do Câncer A.C. Camargo (São Paulo, Brasil) were examined. The age of the patients at surgery ranged from 1 to 208 months (median 42), and there was a predominance of female patients (69.5\%).

The study was approved by the Hospital A.C. Camargo Institutional Ethics Committee.

Immunohistochemical analysis. A representative paraffin block was selected from each case, and $3 \mu \mathrm{m}$-thick sections were prepared for immunohistochemical staining.

Sections were deparaffinized and rehydrated through a graded series of ethanol. Antigen retrieval was performed in a pressure cooker using citrate buffer at a $\mathrm{pH}$ of 6.0. Endogen peroxidase activity was blocked by $3 \%$ perhidrol. Slides were rinsed with PBS (pH 7.4) and incubated at $4^{\circ} \mathrm{C}$ for $18 \mathrm{~h}$ with the primary antibodies (Table I). This was followed by incubation with the respective appropriate secondary antibodies against podoplanin, GLUT-1, ST3, FLI-1, SPARC, endoglin, VEGFA and VEGFC, as well as their receptors (VEGFR1 and VEGFR3) and CD34, CD31 and vWF (factor VIII-related antigen).

StreptABComplex - HRP Duet mouse/rabbit (K0492; Dako) diluted 1:200 in PBS was used as the detection system. Slides were developed in diaminobenzidine solution and counterstained with Harris hematoxylin.

Immunoreactivity was assessed in both vascular endothelial cells and in the stroma of JNA cases by staff pathologists (H.T., H.C. and F.A.S.). The sections were scored according to the degree of positivity observed in the cells. According to our grading scale, cases presenting $<10 \%$ of stained cells were considered negative. Sections incubated without the primary antibody served as negative controls.

RNA isolation and Northern blot analysis. The frozen tissues were pulverized and the total RNA was isolated by the guanidinium isothyocyanate method. Northern blot analysis was performed as previously described (34). An ST3 probe was radiolabeled using a rediprime DNA labeling system with $[\alpha-32$ p]dCTP (Amersham-Pharmacia). The ST3/MMP11 cDNA probe was donated by Dr P. Chambon of the Institute of Genetics and Molecular and Cellular Biology (Strasbourg, France). The autoradiograms were individually quantified by densitometry, and the ratio of each mRNA to 18S mRNA was compared to the average ratio of the controls. The mean value of the expression of the genes in normal turbinates was defined 
Table I. Antibodies used for immunohistochemical analysis.

\begin{tabular}{lclc}
\hline Antibodies & Clones & \multicolumn{1}{c}{ Sources } & Dilutions \\
\hline CD34 & QBEnd 10 & M7165; Dako, Glostrup, Denmark & $1: 100$ \\
CD31 & JC/70A & M0823; Dako & $1: 50$ \\
Factor VIII & Polyclonal & A0082; Dako & $1: 300$ \\
VEGF (A-20) & Polyclonal & sc152; Santa Cruz Biotechnology, Santa Cruz, CA, USA & $1: 600$ \\
GLUT-1 & Polyclonal & A3536; Dako & $1: 500$ \\
Podoplanin & 18H5 & DM3500; Acris antibodies, Hiddenhausen, Germany & $1: 200$ \\
VEGFC & Goat polyclonal & sc1881; Santa Cruz Biotechnology & $1: 100$ \\
ST3/MMP11 & SL3.05 & MS1035P; NeoMarkers, Fremont, CA, USA & $1: 100$ \\
VEGFR3 (FLT4) & Rabbit polyclonal & RB1527; Thermo Scientific, Fremont, CA, USA & $1: 400$ \\
VEGFR1 (Flt-1) & Rabbit polyclonal & RB9255; Thermo Scientific & $1: 100$ \\
FLI-1 & Mouse monoclonal & LLC CM274A,B; Biocare Medical, Concord, CA, USA & $1: 30$ \\
SPARC & Rabbit polyclonal & Ab1858; Chemicon, Temecura, CA, USA & $1: 2,000$ \\
Endoglin & SN6H & MS1290; NeoMarkers & $1: 100$ \\
\hline
\end{tabular}

as the basal or normal expression. An expression of $>2$-fold above that found in normal turbinate tissues was defined as overexpression. Immunohistology was performed to visualize ST3 protein expression in JNAs.

\section{Results}

A total of 22 cases of JNA were included in this study. Results regarding the frequency of positivity of the antibodies utilized are shown in Table II. Podoplanin was evaluated in the JNAs and, although it was evident in the endothelial lining of small lymphatic vessels, was consistently not detected in JNA vessels (Fig. 1B). VEGFC expression was noted in both stromal and endothelial cells in all cases (Fig. 1C). All cases were uniformly negative for VEGFR3 (Fig. 1D). The pan endothelial marker FLI-1 and the marker of activated endothelium, endoglin (CD105), were positive in the vessels in $100 \%$ of the cases. Endoglin was absent in the stromal cells. Single cells also stained positively for FLI-1, but it was not clear whether these cells were endothelial or stromal cells (Fig. 1E and F). SPARC expression was found primarily in the JNA endothelial cells, but was also observed in stellated shaped cells of the stroma (Fig. 1G). Of the 15 cases analyzed by immunohistochemistry, 66.7 and $33.3 \%$ showed ST3 positivity, respectively, in vessels and in numerous single stromal cells (Fig. 1H). Samples of JNA were analyzed for GLUT-1, and the rate of positivity was compared to that found in hemangiomas and in vascular malformation cases. GLUT-1 immunoreactivity was present in the majority of hemangiomas tested (Table III, Fig. 1A), but was absent in vascular malformations and nasofibromas. This difference was highly significant $(\mathrm{p}<0.01)$.

We also evaluated CD31, CD34 and FVIII, which highlighted the vascular endothelium in all cases. CD31 and FVIII were absent in stromal cells, but CD34 showed a weak positivity in $80 \%$ of the stromal cells. VEGFA and VEGFR1 were positive in the endothelial cells in all cases, whereas stromal cells showed a weak expression of both markers in $79 \%$ of the cases (data not shown).
Table II. Frequency of endothelial cell markers in juvenilenasoangiofibromas vessels.

\begin{tabular}{lc}
\hline Markers & Frequency $(\%)$ \\
\hline FLI-1 & $22 / 22(100)$ \\
Endoglin (CD105) & $22 / 22(100)$ \\
Podoplanin & $0 / 22(0)$ \\
VEGFR3 & $0 / 22 \quad(0)$ \\
VEGFC & $22 / 22(100)$ \\
ST3 (MMP11) & $10 / 15(66.7)$ \\
SPARC & $22 / 22(100)$ \\
VEGFA & $21 / 21(100)$ \\
CD31 & $22 / 22(100)$ \\
CD34 & $22 / 22(100)$ \\
FVIII & $22 / 22(100)$ \\
\hline
\end{tabular}

Northern blotting was performed to analyze the mRNA for ST3/MMP11, which was expressed in all tumor samples, showing increased levels as compared to normal turbinates $(0.68 \pm 0.36$ vs. $0.01 \pm 0.006)$ and resulting in significant distribution of these mRNA values $(\mathrm{p}<0.001)$. Representative autoradiographs from Northern blot analysis of ST3 are shown in Fig. 2.

\section{Discussion}

To better characterize the endothelial component of JNAs, our objective in the present study was to determine endothelial proliferation and differentiation markers that have not been previously analyzed in this rare tumor. As it has been suggested that the irregular vascular basement membrane described in JNAs and tumors may possibly result from tissue remodeling (35), we also analyzed the biomarkers of remodeling. 

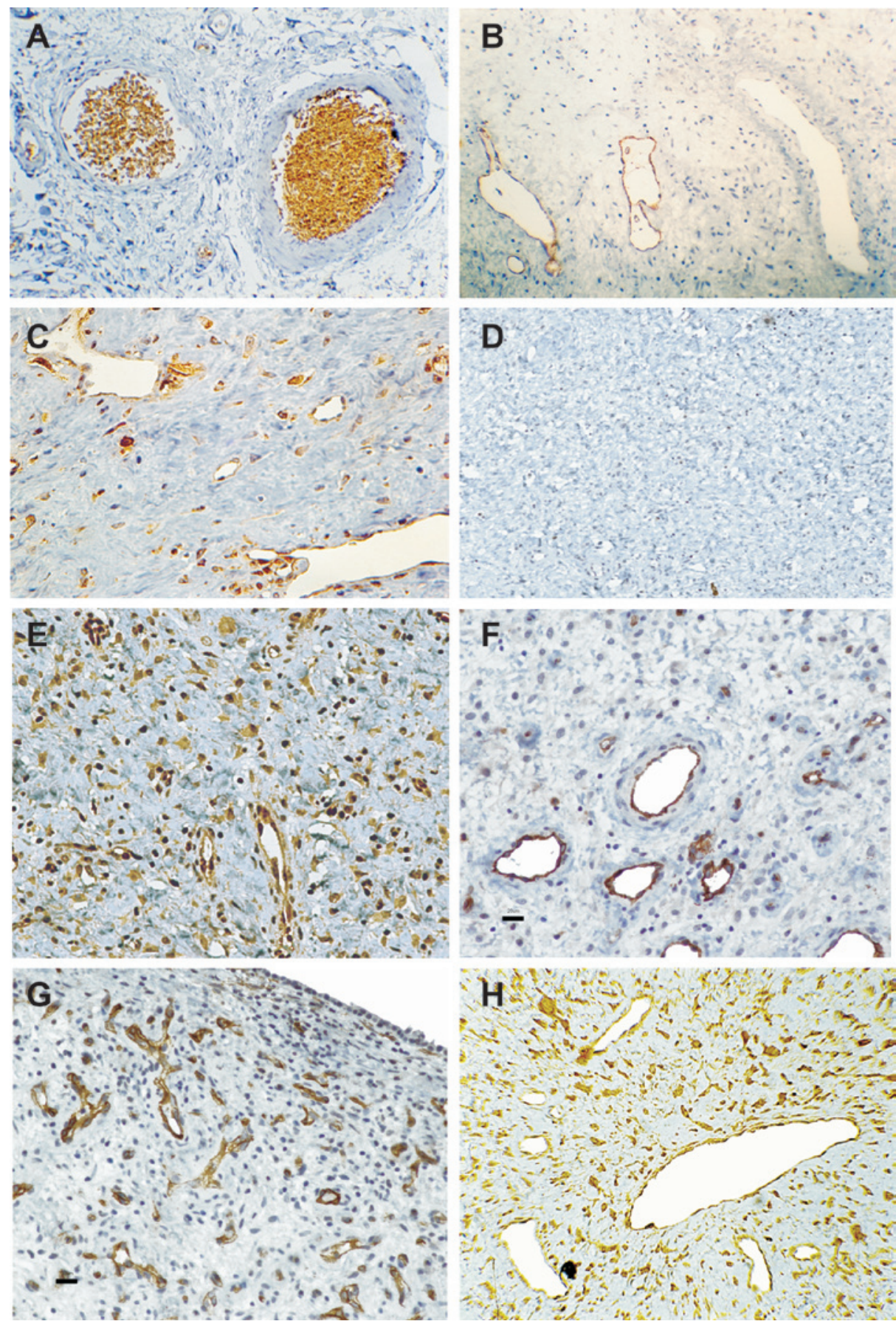

Figure 1. (A) GLUT-1 expressed in the endothelium of infantile proliferating hemangiomas (x200). (B, C, D, E, F, G and H) Immunohistochemical staining of juvenile nasopharyngeal samples for the antibodies used. Podoplanin was evident in the endothelial lining of lymphatic vessels and absent in blood vessels (x200) (B); VEGFC decorated the blood vessels and stromal cells (x200) (C); absence of VEGFR3 staining in JNA vessels (x100) (D); Endothelial cells showing a positive reaction for FLI-1 (x200) (E); endoglin-positive staining in JNA vessels (x200) (F); SPARC expressed by endothelial cells and by a few stromal cells (x200) (G); ST3 positivity in endothelial and stromal cells (x200) (H).

Table III. GLUT-1 immunoreactivity in vascular lesions.

\begin{tabular}{lc}
\hline & GLUT-1 (\%) \\
\hline Vascular malformations & $0 / 135(0)$ \\
Hemangiomas & $101 / 124(81.5)^{\mathrm{a}}$ \\
Angiofibromas & $0 / 22(0)$ \\
\hline
\end{tabular}

aGLUT-1-negative hemangiomas were in an involution phase. Positive cases showed immunoreactivity in more than $10 \%$ of the cells.
Our findings of positive endoglin and Fli-1 staining in the vessels of the cases analyzed here highlight the endothelial differentiation as well as the hyper-proliferative state of angiogenesis in JNAs. Although VEGFC acts primarily as a lymphangiogenic factor, it may also promote the formation of blood vessels (36).

The finding that JNA vessels were devoid of the expression of lymphatic endothelial cell specific markers (podoplanin and VEGFR3) underscores their blood endothelial cell characteristic, without expressing lymphatic competence. Lack of 


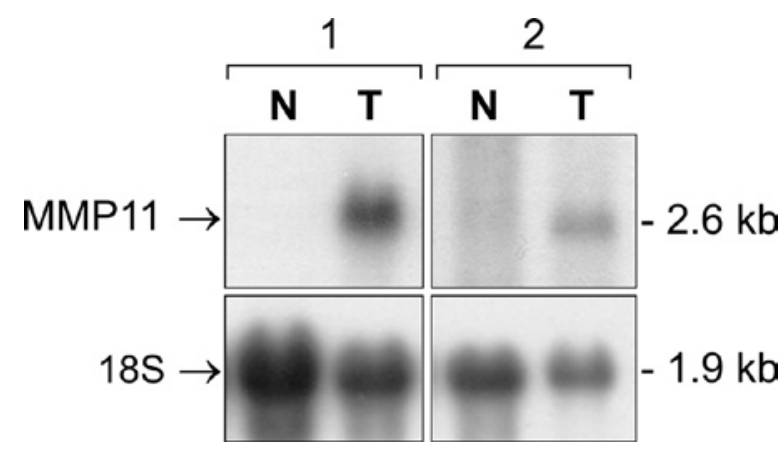

Figure 2. Northern blot analysis of ST3/MMP11 gene expression in JNAs. N, RNA from normal turbinates; T, RNA from JNAs.

GLUT-1 discriminated JNAs from hemangiomas, which is interesting in the context of the suggestion that JNAs might represent vascular malformations $(2,3)$.

Higher levels of ST3 mRNA were found in JNA vessels as compared to inferior nasal turbinate specimens. Although several MMPs have been described in JNAs (37), there have been no reports of ST3 expression in this particular type of tumor. ST3 differs from other MMPs, since its mature form is incapable of cleaving type I and III collagens, the major components of the stromal and vascular ECM in JNAs (7). On the contrary, ST3 exhibits a collagenolytic function against the native $\alpha 3$ chain of collagen VI (38). Prominent collagen-type VI expression has been previously described in JNA vessels, possibly exerting growth stimulatory effects on endothelial cells $(6,7)$.

Several studies reviewed by Clark and Sage (30) indicated that a stressed microenvironment increases the expression of SPARC, reflecting the loss of normal tissue homeostasis. The abundance of SPARC in the late stages of tumor progression and invasion may represent a failed attempt to restore tissue homeostasis within the tumor microenvironment.

According to Chlenski et al (39), SPARC has antiangiogenic properties and is capable of suppressing the activity of VEGF by directly binding to this ligand through a negative regulatory feedback mechanism, as VEGF-induced SPARC (40). Alternatively, SPARC enhances tumor stroma formation, stimulating the production and secretion of several extracellular matrix proteins, including collagen type I, thus favoring the increase of the fibrous component observed during JNA maturation (41). Our results suggest a role for ST3 and SPARC in altering extracellular matrix properties during angiogenesis in JNAs.

Of note, ST3 was reported to be induced by TGF $\beta$ (42), and a reciprocal regulatory loop has been demonstrated for SPARC and TGF $\beta$, since SPARC was shown to induce TGF $\beta$ and vice versa. Endoglin is a type III/TGF $\beta 1$ receptor also up-regulated by TGF $\beta$ stimulation (23), and TGF $\beta$ type II receptor gene transcription is activated by Fli-1 (21). TGF $\beta$ was previously reported in both the stromal and endothelial cells of JNAs $(12,15,16)$. Taken together, our data emphasize the potential role of TGF $\beta 1$ in the pathogenesis of JNA.

\section{Acknowledgements}

This study was supported by CNPq and FAPESP. We thank Dr Waldir Carreirão Neto for help with the collection of samples and the clinical characteristics of the patients and Dr J.P. Plese from the Neurology Department (FM, USP) for the careful review of the manuscript.

\section{References}

1. Coutinho-Camillo CM, Brentani MM and Nagai MA: Genetic alterations in juvenile nasopharyngeal angiofibromas. Head Neck 30: 390-400, 2008.

2. Beham A, Beham-Schmid C, Regauer S, et al: Nasopharyngeal angiofibroma: true neoplasm or vascular malformation? Adv Anat Pathol 7: 36-46, 2000.

3. Schick B and Urbschat S: New aspects of pathogenesis of juvenile angiofibroma. Hosp Med 65: 269-273, 2004.

4. Eivazi B, Ardelean M, Bäumler W, et al: Update on hemangiomas and vascular malformations of the head and neck. Eur Arch Otorhinolaryngol 266: 187-197, 2009.

5. Starlinger V, Wendler O, Gramann M, et al: Laminin expression in juvenile angiofibroma indicates vessel's early developmental stage. Acta Otolaryngol 127: 1310-1315, 2007.

6. Gramann M, Wendler O, Haeberle L and Schick B: Prominent collagen type VI expression in juvenile angiofibromas. Histochem Cell Biol 131: 155-164, 2009.

7. Gramann M, Wendler O, Haeberle L and Schick B: Expression of collagen types I, II and III in juvenile angiofibromas. Cells Tissues Organs 189: 403-409, 2009.

8. Enjolras O, Wassef $\mathrm{M}$ and Chapot R: Introduction: ISSVA Classification. In: Color Atlas of Vascular Tumors and Vascular Malformations. Cambridge University Press, New York, pp3-11, 2007.

9. Tamm E, Jungkunz W, Marsch WC and Lütjen-Drecoll E: Increase in types IV and VI collagen in cherry haemangiomas. Arch Dermatol Res 284: 275-282, 1992.

10. Pusztaszeri MP, Seelentag W and Bosman FT: Immunohistochemical expression of endothelial markers CD31, CD34, von Willebrand factor, and Fli-1 in normal human tissues. J Histochem Cytochem 54: 385-395, 2006.

11. Schiff M, Gonzalez AM, Ong M, et al: Juvenile nasopharyngeal angiofibroma contain an angiogenic growth factor: basic FGF. Laryngoscope 102: 940-945, 1992.

12. Dillard DG, Cohen C, Muller S, et al: Immunolocalization of activated transforming growth factor beta1 in juvenile nasopharyngeal angiofibroma. Arch Otolaryngol Head Neck Surg 126: $723-725,2000$.

13. Zhang PJ, Weber R, Liang $\mathrm{HH}$, et al: Growth factors and receptors in juvenile nasopharyngeal angiofibroma and nasal polyps: an immunohistochemical study. Arch Pathol Lab Med 127: 1480-1484, 2003

14. Brieger J, Wierzbicka M, Sokolov M, et al: Vessel density, proliferation, and immunolocalization of vascular endothelial growth factor in juvenile nasopharyngeal angiofibromas. Arch Otolaryngol Head Neck Surg 130: 727-731, 2004.

15. Saylam G, Yücel OT, Sungur A, et al: Proliferation, angiogenesis and hormonal markers in juvenile nasopharyngeal angiofibroma. Int J Pediatr Otorhinolaryngol 70: 227-234, 2006.

16. Schuon R, Brieger J, Heinrich UR, et al: Immunohistochemical analysis of growth mechanisms in juvenile nasopharyngeal angiofibroma. Eur Arch Otorhinolaryngol 264: 389-394, 2007.

17. Ponti G, Losi L, Pellacani G, et al: Wnt pathway, angiogenetic and hormonal markers in sporadic and familial adenomatous polyposis-associated juvenile nasopharyngeal angiofibromas (JNA). Appl Immunohistochem Mol Morphol 16: 173-178, 2008.

18. Montag AG, Tretiakova M and Richardson M: Steroid hormone receptor expression in nasopharyngeal angiofibromas. Consistent expression of estrogen receptor beta. Am J Clin Pathol 125: 832-837, 2006.

19. Pauli J, Gundelach R, Vanelli-Rees A, et al: Juvenile nasopharyngeal angiofibroma: an immunohistochemical characterisation of the stromal cell. Pathology 40: 396-400, 2008.

20. Nagy JA, Chang SH, Dvorak AM and Dvorak HF: Why are tumour blood vessels abnormal and why is it important to know? Br J Cancer 100: 865-869, 2009.

21. Truong AH and Ben-David Y: The role of Fli-1 in normal cell function and malignant transformation. Oncogene 19: 6482-6489, 2000.

22. Folpe AL, Chand EM, Goldblum JR and Weiss SW: Expression of Fli-1, a nuclear transcription factor, distinguishes vascular neoplasms from potential mimics. Am J Surg Pathol 25: 1061-1066, 2001. 
23. Dallas NA, Samuel S, Xia L, Fan F, Gray MJ, Lim SJ and Ellis LM: Endoglin (CD105): a marker of tumor vasculature and potential target for therapy. Clin Cancer Res 14: 1931-1937, 2008.

24. Breiteneder-Geleff S, Soleiman A, Kowalski H, et al: Angiosarcomas express mixed endothelial phenotypes of blood and lymphatic capillaries: podoplanin as a specific marker for lymphatic endothelium. Am J Pathol 154: 385-394, 1999.

25. Folpe AL, Veikkola T, Valtola R and Weiss SW: Vascular endothelial growth factor receptor-3 (VEGFR-3): a marker of vascular tumors with presumed lymphatic differentiation, including Kaposi's sarcoma, kaposiform and Dabska-type hemangioendotheliomas, and a subset of angiosarcomas. Mod Pathol 13: 180-185, 2000.

26. Harik SI, Hall AK, Richey P, et al: Ontogeny of the erythroid/ HepG2-type glucose transporter (GLUT-1) in the rat nervous system. Brain Res Dev Brain Res 72: 41-49, 1993.

27. Gillies RJ, Robey I and Gatenby RA: Causes and consequences of increased glucose metabolism of cancers. J Nucl Med 49: S24-S42, 2008.

28. North PE, Waner M, Mizeracki A, et al: GLUT-1: a newly discovered immunohistochemical marker for juvenile hemangiomas. Hum Pathol 31: 11-22, 2000.

29. Aird WC: Molecular heterogeneity of tumor endothelium. Cell Tissue Res 335: 271-281, 2009

30. Clark CJ and Sage EH: A prototypic matricellular protein in the tumor microenvironment - where there's SPARC, there's fire. J Cell Biochem 104: 721-732, 2008.

31. Rio MC: From a unique cell to metastasis is a long way to go: clues to stromelysin-3 participation. Biochimie 87: 299-306, 2005.

32. Podhajcer OL, Benedetti L, Girotti MR, Prada F, Salvatierra E and Llera AS: The role of the matricellular protein SPARC in the dynamic interaction between the tumor and the host. Cancer Metastasis Rev 27: 523-537, 2008.

33. Krstulja M, Car A, Bonifacić D, Braut T and Kujundzić M: Nasopharyngeal angiofibroma with intracellular accumulation of SPARC - a hypothesis (SPARC in nasopharyngeal angiofibroma). Med Hypotheses 70: 600-604, 2008.
34. Mangone FR, Brentani MM, Nonogaki S, et al: Overexpression of Fos-related antigen-1 in head and neck squamous cell carcinoma. Int J Exp Pathol 86: 205-212, 2005.

35. Baluk P, Morikawa S, Haskell A, Mancuso M and McDonald DM: Abnormalities of basement membrane on blood vessels and endothelial sprouts in tumors. Am J Pathol 163: 1801-1815, 2003.

36. Cao R, Eriksson A, Kubo H, Alitalo K, Cao Y and Thyberg J: Comparative evaluation of FGF-2-, VEGF-A-, and VEGF-Cinduced angiogenesis, lymphangiogenesis, vascular fenestrations, and permeability. Circ Res 94: 664-670, 2004.

37. Duerr S, Wendler O, Aigner T, Karosi S and Schick B Metalloproteinases in juvenile angiofibroma - a collagen rich tumor. Hum Pathol 39: 259-268, 2008.

38. Motrescu ER, Blaise S, Etique N, et al: Matrix metalloproteinase-11/stromelysin-3 exhibits collagenolytic function against collagen VI under normal and malignant conditions. Oncogene 27: 6347-6355, 2008.

39. Chlenski A, Liu S, Guerrero LJ, et al: SPARC expression is associated with impaired tumor growth, inhibited angiogenesis and changes in the extracellular matrix. Int J Cancer 118: 310-316, 2006.

40. Kato Y, Lewalle JM, Baba Y, et al: Induction of SPARC by VEGF in human vascular endothelial cells. Biochem Biophys Res Commun 287: 422-426, 2001.

41. Sennes LU, Fortes FS, Butugan O, Saldiva PH and Bernardi FC: Tissue maturation correlating to clinical manifestations in juvenile angiofibroma. Ann Otol Rhinol Laryngol 114: 705-708, 2005.

42. Delany AM and Canalis E: The metastasis-associated metalloproteinase stromelysin-3 is induced by transforming growth factor-beta in osteoblasts and fibroblasts. Endocrinology 142 1561-1566, 2001 